\title{
The prognostic utility and clinical outcomes of MNX1-AS1 expression in cancers: a systematic review and meta-analysis
}

Juan Li

The first affiliated hospital, college of medicine, zhejiang university https://orcid.org/0000-0002-0121-7098

Wen Jin

The first affiliated hospital, college of medicine, zhejiang university

Zhengyu Zhang

The first affiliated hospital, college of medicine, zhejiang university

Jingjing Chu

The first affiliated hospital, college of medicine, zhejiang university

Hui Yang

The first affiliated hospital, college of meicine, zhejiang university

\section{Chang Li}

the first affiliated hospital, college of medicine, zhejiang university

Ruiyin Dong

The first affiliated hospital, college of medicine, zhejiang university

Cailian Zhao ( $\nabla$ clz1126@126.com)

https://orcid.org/0000-0001-8337-0610

Primary research

Keywords: Long non-coding RNA, MNX1-AS1, Cancer, Prognosis

Posted Date: March 25th, 2020

DOl: https://doi.org/10.21203/rs.3.rs-19089/v1

License: @) (1) This work is licensed under a Creative Commons Attribution 4.0 International License. Read Full License 


\begin{abstract}
Background: Recently, emerging studies have identified that MNX1-AS1 highly expressed among variety of cancers and related with worse prognosis of cancer patients. The purpose of this study was to evaluate the relationship between MNX1-AS1 expression with clinical features and prognosis in different cancers.
\end{abstract}

Methods: In this study, we searched the Web of Science, PubMed, CNKI, and Wanfang databases to find relevant studies of MNX1-AS1. Pooled hazard ratios (HRs) and odds ratios (ORs) with 95\% confidence intervals (Cls) were applied to explore the prognostic and clinical significance of MNX1-AS1.

Results: A total of 9 literatures were included in this study, including 882 cancer patients. The results showed that patients with increased MNX-AS1 expression were more likely to develop lymph node metastasis (OR $=5.616,95 \% \mathrm{Cl}: 3.093-10.199, \mathrm{P}=0.000)$ and advanced TNM stage $(\mathrm{OR}=4.625,95 \% \mathrm{Cl}: 2.366-9.040$, $\mathrm{P}=0.000)$. Moreover, we demonstrated that patients with high expression of MNX1-AS1 had poor OS in different cancers (HR = 1.976, $95 \% \mathrm{Cl}$ : $1.653-2.361$, $\mathrm{P}=0.000)$. In addition, patients with high expression of MNX1-AS1 suffer from worse prognosis in gastric cancer $(\mathrm{HR}=2.385,95 \% \mathrm{Cl}: 1.838-3.094, \mathrm{P}=0.000)$ and lung cancer $(\mathrm{HR}=1.959,95 \% \mathrm{Cl}: 1.353-2.835, \mathrm{P}=0.000)$.

Conclusions: High MNX1-AS1 expression is significantly associated with unfavorable clinical outcomes and it has the potential to serve as a prognostic biomarker in cancer patients.

\title{
Background
}

Long non-coding RNA (LncRNA) is a novel type of non-coding RNA with longer than 200 nucleotides, and has the characteristics of lacking protein-coding capacity and open reading frames[1-3]. Growing evidence from recent studies has indicted that in different oncogenic signaling pathways, IncRNAs mediate multiple molecular mechanisms, including alternative splicing of precursor mRNAs, functions of competing for endogenous RNA, promotion of epithelialmesenchymal transition (EMT), epigenetic modification and cells phenotypic changes, etc., and finally promote the occurrence and progression of malignant tumors $[1,2,4,5]$.

Motor neuron and pancreas homeobox 1-antisense RNA1 (MNX1-AS1), is the antisense strand of MNX1 (7q36.6), also known as MAYA and CCAT5, which maps to chromosome 7q36.3[6, 7]. Several studies for the first time provided that MNX1-AS1 has a sponge adsorption effect on miR-6785-5p, thereby upregulating the expression of BCL2, and overexpression of MNX1-AS1 might facilitate the proliferation, migration and invasion of gastric cancer cells [8-10]. Knockdown of MNX1-AS1 might affect the expression of CDK4, cyclin D, Bax, and Bcl-2, which may inhibit proliferation and migration capabilities, and promote apoptosis of ovarian cancer cells[11]. In recent years, some studies have reported that MNX1-AS1 is upregulated in tissues of various cancers, including: hepatocellular carcinoma[12], bladder Cancer[13], esophageal squamous cell carcinoma[14, 15], lung cancer[16], prostate cancer[17], cervical cancer[18], etc. As a result, these evidences indicated that MNX1-AS1 overexpression may play a pivotal role in predicting worse or favorable prognosis of cancer patients. In addition, numerous independent studies have investigated the prognostic performance of MNX1-AS1 expression among various cancers. However, most published findings were limited by sample size and research methods. Therefore, it is imperative to conduct a comprehensive meta-analysis to systematically assess relationship of MNX1-AS1 expression with patient clinical characteristics and prognosis in different malignant cancers by reviewing all related published studies.

\section{Materials And Methods Retrieval Strategy}

This study searched the Web of Science, PubMed, CNKI, and Wanfang databases for getting related articles up to January 31,2020 . Search by matching the following keywords: IncRNA, MNX1-AS1, cancer, tumor, malignancy, neoplasm, lymphoma, leukemia. Two researchers (JL and ZYZ) screened strictly the related literatures according to the inclusion and exclusion criteria.

\section{Inclusion And Exclusion Criteria}

Inclusion criteria: (a) the original study used qRT-PCR to detect the expression of MNX1-AS1. (b) cancer patients was divided into both high and low expression groups according to expression level of MNX1-AS1. (c) the study provided the relationship between overall survival (OS) or clinicopathological parameters and MNX1-AS1 expression. (d) all patients of the original study were confirmed by histopathology. (e) sample size of the eligible study more than 40.

Exclusion criteria: (a) the study did provide Kaplan-Meier survival curves. (b) incomplete data made it impossible to obtain hazard ratio (HR) data. (c) sample size of the original study less than 40 . (d) non-human research, letters, case reports, meta-analysis, review articles and other studies.

\section{Quality Evaluation And Data Extraction}

Two investigator (JL and ZYZ) evaluated the quality of the included literature independently in accordance with quality evaluation criteria of NewcastleOttawa (NOS). The qualitied articles with NOS $\geq 6$ scores were ultimately regard as high-quality or eligible studies. The contents of the data extraction are as follows: first author, publication year, country, number of cases, and patient clinical parameters including: age, gender, TNM stage, differentiation, distant metastasis (DM), tumor size and lymph node metastasis (LNM), overall survival rate, HR (hazard ratio) and $95 \% \mathrm{Cl}$. Engauge Digitizer v4.1 software extracted data of Kaplan-Meier curve to obtaining HR and $95 \% \mathrm{Cl}$ by the study of Tierney et al. [19]. Two researchers (JL and ZYZ) extracted the relevance data independently. When they encounter disagreements, they would discuss with a third researcher $(\mathrm{WJ})$ to decide the final results.

Page 2/9 


\section{Statistical analysis}

The odds ratios (ORs) and hazard ratios (HRs) and their 95\% confidence intervals (Cls) were combined to quantitatively evaluate the correlation of MNX1-AS1 expression with clinical parameters and prognostic implication of cancer. The heterogeneity across the studies was assessed by using Cochrane Q-test and ${ }^{2}$ statistics. For $P>.05$ as well as $\mathrm{I}^{2}<50 \%$, the fixed-effect model was adopted to pool the related results. Otherwise, the random-effect model was chosen. Sensitivity analysis assessed the stability of pooled HRs/ORs value. Begg and Egger tests, as well as funnel plots were used to appraise publication bias. All P values less than 0.05 was considered statistically significant. Statistical analysis was performed using Stata 11 software.

\section{Results}

\section{Search results of eligible studies}

After preliminary retrieval, we retrieved 44 literatures in total from online databases. We did not obtain the related studies among Chinese databases. Ultimately, 9 articles were contained in the current study. The selection process of 9 eligible articles presented in Fig. 1. A total of 882 patients from the all eligible articles were included to evaluate prognostic value of MNX1-AS1 (presented in Table 1). All articles covered gastric cancer(GC) $(n=3)$ [8-10], lung cancer $(L C)(n=3)[6,20,21]$, other cancer $(n=3)$ including: esophageal squamous cell carcinoma (ESCC), cervical cancer (CC), epithelial ovarian cancer $(E O C)$ $[14,18,22]$.

Table 1

Characteristics of the included studies

\begin{tabular}{|c|c|c|c|c|c|c|c|c|}
\hline First author & Year & Type of cancers & Detection method & Follow-up(months) & Cut-off value & Sample size & Sources of HR & Survival outcome \\
\hline Chu J[1] & 2019 & ESCC & qRT-PCR & 120 & Median & 46 & K-M Curve & os \\
\hline Liu G[2] & 2019 & NSCLC & qRT-PCR & 60 & Median & 116 & K-M Curve & os \\
\hline Zhang W[3] & 2019 & $\mathrm{GC}$ & qRT-PCR & 60 & Median & 96 & Original paper & os \\
\hline Liu H[4] & 2019 & LC & qRT-PCR & 60 & Median & 43 & K-M Curve & os \\
\hline Yang R[5] & 2018 & LAC & qRT-PCR & 96 & Mean & 124 & Original paper & os \\
\hline Liu X[6] & 2019 & $\mathrm{CC}$ & qRT-PCR & 60 & Median & 54 & K-M Curve & os \\
\hline Shuai Y[7] & 2020 & $\mathrm{GC}$ & qRT-PCR & 40 & Median & 174 & K-M Curve & OS/DFS \\
\hline Li A[8] & 2017 & EOC & qRT-PCR & 60 & Median & 177 & Original paper & OS/PFS \\
\hline Ma J[9] & 2019 & $\mathrm{GC}$ & qRT-PCR & 60 & Median & 52 & K-M Curve & os \\
\hline
\end{tabular}

\section{Relationship Of MNX1-AS1 Expression With Clinical Characteristics}

The summary of SNHG12 expression and clinical features presented Table 2. As shown in Table 3, pooled analyses showed that MNX1-AS1 expression did not correlate significantly with age or gender, which implied that the original study gender and age were comparable. The heterogeneity among studies was not statistically significant (Age: $\mathrm{I}^{2}=0.0 \%, \mathrm{P}=0.888$; Gender: $\mathrm{I}^{2}=0.0 \%, \mathrm{P}=0.696$ ). Compared with the gene low expression, cancer patients with high expression of MNX1-AS1 had a 4.081-fold increased risk of lymph node metastasis (OR = 5.616, 95\% Cl: 3.093-10.1991, P=0.000, I-squared = 50.4, P = 0.073). The relationship between MNX1-AS1 expression and clinical stage was statistically significant $(\mathrm{OR}=4.625,95 \% \mathrm{Cl}$ : $2.366-9.040, \mathrm{P}<0.001)$. 
Table 2

The summary of MNX1-AS1 expression and clinical features

\begin{tabular}{|c|c|c|c|c|c|c|c|c|c|c|c|c|c|c|c|c|c|c|c|c|c|c|c|c|}
\hline \multirow{2}{*}{$\begin{array}{l}\text { Characteristics } \\
\text { Study ID }\end{array}$} & \multicolumn{4}{|c|}{$\begin{array}{l}\text { Age (elderly vs. } \\
\text { nonelderly) }\end{array}$} & \multicolumn{4}{|c|}{$\begin{array}{l}\text { Gender (female vs. } \\
\text { male) }\end{array}$} & \multicolumn{4}{|c|}{$\begin{array}{l}\text { Tumor size }(\mathrm{cm}) \\
\text { (large size vs. small } \\
\text { size) }\end{array}$} & \multicolumn{4}{|c|}{$\begin{array}{l}\text { Lymph node } \\
\text { metastasis (positive } \\
\text { vs. negative) }\end{array}$} & \multicolumn{4}{|c|}{$\begin{array}{l}\text { Distant metastasis } \\
\text { (presence vs. } \\
\text { absence) }\end{array}$} & \multicolumn{4}{|c|}{$\begin{array}{l}\text { Differentiation (po } \\
\text { vs. well, moderate) }\end{array}$} \\
\hline & a1 & $\mathrm{a} 0$ & b1 & b0 & a1 & $\mathrm{a} 0$ & b1 & b0 & a1 & $\mathrm{a} 0$ & b1 & b0 & a1 & $\mathrm{a} 0$ & b1 & b0 & a1 & $\mathrm{a} 0$ & b1 & b0 & a1 & $\mathrm{a} 0$ & b1 & [ \\
\hline Chu J[1] & 12 & 11 & 14 & 8 & 10 & 13 & 12 & 10 & 16 & 7 & 10 & 12 & 19 & 4 & 3 & 19 & & & & & 6 & 16 & 6 & 1 \\
\hline Liu G[2] & 38 & 20 & 37 & 21 & 26 & 32 & 20 & 38 & & & & & 51 & 7 & 24 & 34 & & & & & 36 & 22 & 31 & 2 \\
\hline Zhang W[3] & 25 & 23 & 31 & 17 & 19 & 29 & 23 & 25 & 33 & 15 & 13 & 35 & 31 & 17 & 13 & 35 & & & & & 31 & 17 & 36 & 1 \\
\hline Liu H[4] & 15 & 5 & 15 & 8 & 3 & 17 & 5 & 18 & & & & & 16 & 4 & 10 & 13 & & & & & & & & \\
\hline Yang R[5] & 36 & 26 & 38 & 24 & 21 & 41 & 20 & 42 & & & & & & & & & 15 & 47 & 0 & 62 & 32 & 30 & 1 & $\angle$ \\
\hline Liu X[6] & 11 & 18 & 12 & 13 & & & & & 22 & 7 & 8 & 17 & 18 & 11 & 8 & 17 & & & & & 21 & 8 & 15 & 1 \\
\hline Shuai Y[7] & 73 & 14 & 78 & 9 & 20 & 67 & 23 & 64 & 35 & 52 & 54 & 33 & 69 & 18 & 50 & 37 & 11 & 76 & 2 & 85 & 58 & 28 & 45 & $\angle$ \\
\hline Li A[8] & 40 & 47 & 49 & 41 & & & & & 30 & 57 & 23 & 67 & & & & & 44 & 43 & 69 & 21 & 50 & 37 & 65 & \\
\hline
\end{tabular}

a1, a0 (ref): the number of MNX1-AS1 high expression; b1, b0 (ref): the number of low expression of MNX1-AS1.

Table 3

Association between MNX1-AS1 and clinical parameters

\begin{tabular}{|lllllll|}
\hline Clinical parameters & Studies(n) & Cases(n) & OR(95\%Cl) & P & Heterogeneity $\left(\mathbf{I}^{2}, \mathbf{P}\right)$ & Model \\
\hline Age (elderly vs. nonelderly) & 8 & 829 & $0.774(0.576-1.040)$ & 0.089 & $0.0 \%, 0.888$ & Fixed \\
\hline Gender (female vs. male) & 6 & 598 & $0.941(0.667-1.328)$ & 0.730 & $0.0 \%, 0.696$ & Fixed \\
\hline Tumor size (non-small vs. small) & 5 & 546 & $2.202(0.735-6.599)$ & 0.159 & $88.1 \%, 0.000$ & Random \\
\hline Lymph node metastasis (N1 vs. N0) & 6 & 528 & $5.616(3.093-10.199)$ & 0.000 & $50.4 \%, 0.073$ & Random \\
\hline Distant metastasis(M1 vs. M0) & 3 & 475 & $3.480(0.165-73.384)$ & 0.423 & $91.9 \%, 0.000$ & Random \\
\hline Differentiation (poor vs. well, moderate) & 7 & 785 & $1.242(0.739-2.090)$ & 0.413 & $64.9 \%, 0.009$ & Random \\
\hline TNM stage (III + IV vs. I+ II) & 7 & 775 & $4.625(2.366-9.040)$ & 0.000 & $75 \%, 0.001$ & Random \\
\hline
\end{tabular}

\section{Relationship Of MNX1-AS1 Expression With Prognosis}

This study showed that the high expression of MNX1-AS1 was significantly related to short overall survival time of cancer patients (HR $=1.976,95 \%$ Cl: $1.653-2.361, P=0.000$, I-squared $=28.0 \%$ ), shown in Fig. 2A. Subgroup analyses based on the type of cancer provided that the $0 S$ rate of patients among gastric cancer with high expression of MNX1-AS1 lower than that of patients with low expression of MNX1-AS1 (HR=2.385, 95\% Cl: 1.838-3.094, P = 0.000). In addition, similar results appeared in patients with lung cancer $(\mathrm{HR}=1.959,95 \% \mathrm{Cl}: 1.353-2.835, \mathrm{P}=0.000)$. Significant heterogeneity was not found in the GC (I-squared $=0.0 \%, P=0.943)$, and LC (I-squared $=0.0 \%, P=0.508)$ subgroups. Subgroup analyses of sample size identified that the expression of MNX1AS1 was associated with prognosis of cancer patients (sample size $\geq 100: \mathrm{HR}=2.141, \mathrm{P}=0.000$, fixed effect model; sample size < 100: $\mathrm{HR}=1.836, \mathrm{P}=0.006$, random effect model), presented in Fig. 2B. Unlike sample size less than 100 ( $\mathrm{l}$-squared $=53.8 \%, P=0.070$ ), no significant heterogeneity was found in subgroups of sample size greater than 100 (sample size $\geq 100$ : I-squared $=0.0 \%, P=0.559$ ).

\section{Publish Bias And Sensitivity Analysis}

In this study, funnel plot, Egger regression and Begg rank correlation tests were used to evaluate potential publication bias. As shown in Table S1, there was no publication bias among studies on clinical parameters. Begg and Egger tests also demonstrated that no significant publication bias was found for OS (Egger's test: $P=0.058$; Begg's test: $P=0.150$ ), and the corresponding funnel plot is shown in Fig. 3 . The results of sensitivity analysis provided that after excluding any of the studies, the value of the combined HRs or ORs did not change significantly, indicating that the pooling results were stability and robustness.

\section{Involvement Of MNX1-AS1 In Carcinogenesis}

In order to further discover roles of MNX1-AS1 in cancer, we elaborated the effects of MNX1-AS1 on prognostic biomarker and cell phenotype, as well as MNX1-AS1-mediated carcinogenesis, as presented in Table 4. MNX1-AS1 induced Epithelial-mesenchymal transition (EMT) by virtue of promoting N-cadherin and vimentin, and inhibiting E-cadherin[7, 17]. Moreover, MNX1-AS1 could competitively bind to miRNA to regulate related gene or protein expression[13, 23]. 
Additionally, MNX1-AS1 also activates oncogenic pathways including MAPK, BRF2, and AKT/mTOR pathways[7, 18, 21]. Taken together, MNX1-AS1 usually regulates with other relevant genes or proteins and cooperates to exert oncogenic functions through involving the carcinogenic signaling pathways.

Table 4

Research discoveries of MNX1-AS1 among cancers ( $\uparrow$ represents increase; $\downarrow$ represents decrease)

\begin{tabular}{|c|c|c|c|c|c|}
\hline Cancer type & $\begin{array}{l}\text { Expression } \\
\text { effect }\end{array}$ & $\begin{array}{l}\text { Clinical and } \\
\text { prognostic features } \\
\text { of MNX1-AS1 }\end{array}$ & $\begin{array}{l}\text { Transition of cell phenotype of } \\
\text { upregulated MNX1-AS1 }\end{array}$ & Carcinogenic mechanisms & References \\
\hline Gastric cancer & upregulation & $\begin{array}{l}\uparrow L N M / D M / \text { tumor } \\
\text { size/TNM stage; } \\
\text { \Differentiation; } \\
\text { \OS/DFS }\end{array}$ & $\begin{array}{l}\text { pproliferation/migration/invasion; } \\
\text { \apoptosis; } \downarrow \text { G1/G0 phase arrest }\end{array}$ & $\begin{array}{l}\text { TEAD4 modulated MNX1-AS1, which } \\
\text { suppressed BTG2 through EZH2-mediated } \\
\text { H3K27me3; MNX1-AS1 functions as a } \\
\text { ceRNA and sponges miR-6785-5p to } \\
\text { regulate BCL2; MNX1-AS1 regulated } \\
\text { PCNA, EMT-related markers (N-cadherin } \\
\text { and vimentin), MMP-9, and CDKN1A. }\end{array}$ & {$[8-10]$} \\
\hline Lung cancer & upregulation & $\begin{array}{l}\uparrow D M / L N M / \text { tumor } \\
\text { size/TNM stage; } \\
\downarrow \text { Differentiation; } \downarrow \text { OS }\end{array}$ & $\begin{array}{l}\uparrow \text { proliferation/migration/invasion; } \\
\downarrow \text { apoptosis }\end{array}$ & $\begin{array}{l}\text { MNX1-AS1 facilitated BRF2 by inhibiting } \\
\text { miR-527; MNX1-AS1 regulated BCL-2, Bax, } \\
\text { MMP-2, MMP-9; MNX1-AS1 silencing } \\
\text { promoted E-cadherin and inhibited N- } \\
\text { cadherin/vimentin to regulate EMT. }\end{array}$ & {$[6,20,21]$} \\
\hline $\begin{array}{l}\text { Esophageal } \\
\text { squamous cell } \\
\text { carcinoma }\end{array}$ & upregulation & $\uparrow L N M$ & $\begin{array}{l}\uparrow \text { proliferation/migration/invasion; } \\
\text { Japoptosis; Icycle arrest in G1 } \\
\text { phase; } \uparrow \text { cycle arrest in S phase }\end{array}$ & $\begin{array}{l}\text { MNX1-AS1 functioned as ceRNA for miR- } \\
\text { 34a; MNX1-AS1 regulated SIRT1 } \\
\text { expression via miR-34a. MNX1-AS1 } \\
\text { regulated miR-34a/SIRT1 axis. }\end{array}$ & {$[14,15]$} \\
\hline Glioblastoma & upregulation & - & $\uparrow$ proliferation/migration/invasion & $\begin{array}{l}\text { MNX1-AS1 acted as a sponge of miR- } \\
4443 \text {. }\end{array}$ & [25] \\
\hline $\begin{array}{l}\text { Hepatocellular } \\
\text { carcinoma }\end{array}$ & upregulation & १TNM stage; $\downarrow$ OS & $\uparrow$ proliferation/migration/invasion & $\begin{array}{l}\text { MNX1-AS1 promoted COMMD8 } \\
\text { expression by sponging miR-218-5p. }\end{array}$ & [12] \\
\hline Ovarian cancer & upregulation & $\begin{array}{l}\uparrow F I G O \\
\text { stage/LNM/DM; } \\
\downarrow \text { OS/PFS }\end{array}$ & $\begin{array}{l}\text { iproliferation/colony formation/ } \\
\text { migration; } \downarrow \text { cycle arrest at the } \\
\text { G0/G1 phase; } \downarrow \text { apoptosis }\end{array}$ & $\begin{array}{l}\text { MNX1-AS1 knockdown could alter the } \\
\text { protein expressions of CDK4, cyclin D, } \\
\text { Bax, and Bcl-2. }\end{array}$ & {$[11,22]$} \\
\hline Cervical cancer & upregulation & $\begin{array}{l}\uparrow \text { LNM/FIGO stage } \\
\text { /tumor } \\
\text { size/vascular } \\
\text { invasion; } \downarrow \text { OS }\end{array}$ & $\uparrow$ proliferation; $\downarrow$ apoptosis & $\begin{array}{l}\text { MNX1-AS1 improved the expression of p- } \\
\text { ERK1/2 and p-JNK; MNX1-AS1 activated } \\
\text { MAPK pathway. }\end{array}$ & [18] \\
\hline Breast cancer & upregulation & - & $\uparrow$ proliferation/migration/invasion & $\begin{array}{l}\text { MNX1-AS1 induced EMT and activated } \\
\text { the AKT/mTOR pathway; MNX1-AS1 } \\
\text { could promote the expression of EMT- } \\
\text { related proteins; MNX1-AS1 regulated } \\
\text { CDK4, BCL2, cyclinD1 and c-MYC. }\end{array}$ & [7] \\
\hline Bladder Cancer & upregulation & - & $\uparrow$ proliferation/migration/invasion & $\begin{array}{l}\text { MNX1-AS1 could competitively bind to } \\
\text { miR-218-5p to regulate RAB1A. }\end{array}$ & [13] \\
\hline Prostate cancer. & upregulation & - & $\uparrow$ proliferation/migration/invasion & $\begin{array}{l}\text { Knockdown of MNX1-AS1 promoted E- } \\
\text { cadherin expression whereas it inhibited } \\
\text { N-cadherin, vimentin, PCNA, PH-3 } \\
\text { expression. }\end{array}$ & [17] \\
\hline $\begin{array}{l}\text { Colon } \\
\text { adenocarcinoma }\end{array}$ & upregulation & - & $\uparrow$ proliferation/migration/invasion & $\begin{array}{l}\text { MNX1-AS1 interacts with E2F1; MNX1- } \\
\text { AS1 could sponge microRNA-218-5p to } \\
\text { upregulate SEC61A1. }\end{array}$ & [23] \\
\hline
\end{tabular}

\section{Discussion}

MNX1-AS1, also known as MAYA and CCAT5, is located at chromosome 7q36.3[6]. Upregulation of MNX1-AS1 expression has been found in a variety of cancers, including gastric cancer[8-10], lung cancer[6, 20, 21], esophageal squamous cell carcinoma[14, 15], and estrogen-dependent cancer[7, 18, 22], etc. MNX1-AS1 play a role of functional oncogene in addition to activating AKT/mTOR pathway and its natural sense transcript MNX1 in breast cancer cells, it also promotes the process of EMT[7]. MNX1-AS1 functions as a sponge to miR-218-5p to regulate expression of RAB1A, which indicated that MNX1-AS1 might serve as a particularly novel biomarker for migration, invasion, proliferation, and EMT of bladder cancer[13]. Furthermore, MNX1-AS1 promotes BRF2 expression by inhibiting activity of miR-527, might facilitate proliferation, migration, and invasion of lung cancer[24]. MNX1-AS1 also induced proliferation and repressed apoptosis of cervical cancer cells through MAPK signaling pathway[18]. Overall, MNX1-AS1 might facilitate carcinogenesis of different cancer by exerting its carcinogenic molecular function to regulate the expression of miRNA and other related genes or protein and activate the corresponding signal pathway[23, 25]. Therefore, future research can deeply explore the potential of MNX1-AS1 as a biomarker, which is valuable for evaluating prognosis, early diagnosis, and targeted therapy of different cancers.

In recent years, several published studies reported that the association of MNX1-AS1 expression with prognosis, tumor size, lymph node metastasis, distant metastasis, differentiation, as well as TNM stage. Consequently, we performed a meta-analysis with the goal of comprehensively assessing the prognostic value of MNX1-AS1. The study is the first study on the expression of MNX1-AS1 and the prognosis of cancer. The combined results presented that patients 
with MNX1-AS1 over-expression were susceptible to suffer from unfavorable prognosis in gastric cancer and lung cancer. The pooling results of the study are consistent with most of the original literatures, which recommend that MNX1-AS1 high expression might be associated with worse prognosis of malignant cancer patients[6, 8, 9]. Moreover, the sensitivity analysis proved the stability and reliability of the pooled results. The heterogeneity of gastric cancer (Isquared $=0.0 \%, P=0.943$ ), lung cancer (I-squared $=0.0 \%, P=0.508)$, and sample sizes $\geq 100$ (I-squared $=0.0 \%, 0.559)$ subgroups was lower than the overall heterogeneity in the study. Therefore, subgroup analysis of this study demonstrated that the heterogeneity among the included studies stems from cancer type and sample size. Additionally, MNX1-AS1 expression was significantly related to lymph node metastasis and TNM stage. However, further studies on the possible relationship of MNX1-AS1 expression with tumor size, distant metastasis or differentiation are needed. Therefore, based on the above evidence, MNX1-AS1 may be a novel candidate biomarker of cancer prognosis.

There are several limitations in the study. First, the cut-off values of MNX1-AS1 expression were different across studies. Second, cancer type and sample size might explain source of heterogeneity. Finally, HRs and $95 \% \mathrm{Cls}$ of several studies were extracted from the Kaplan-Meier curves. Therefore, larger-scale welldesigned studies are needed to confirm the result of this study.

\section{Conclusions}

In conclusion, we indicated that high MNX1-AS1 expression is associated with unfavorable OS and poor clinical outcomes in patients with different types of cancer.

\section{Abbreviations}

LncRNAs: long noncoding RNAs; MNX1-AS1: Motor neuron and pancreas homeobox 1-antisense RNA1; ESCC: esophageal squamous cell carcinoma; NSCLC: non-small cell lung cancer;GC: gastric cancer; LC:lung cancer; LAC:lung adenocarcinoma; CC: cervical cancer; EOC: epithelial ovarian cancer; LNM: lymph node metastasis; DM: Distant metastasis; ceRNA: competitive endogenous RNA.

\section{Declarations}

\section{Authors' contributions}

$\mathrm{JL}$ conceived and wrote the entire paper. JL and ZYZ collected and analyzed the related data. WJ, JJC, HY, RYD, CL and CLZ revised the paper. All authors have reviewed the final version of the manuscript.

\section{Author details}

${ }^{1}$ The First Affiliated Hospital, College of Medicine, Zhejiang University.

\section{Acknowledgements}

Not applicable.

\section{Competing interests}

All authors proclaimed no conflicts of interest in the current study.

\section{Availability of data and materials}

Please contact authors for all available data.

\section{Consent for publication}

Not applicable.

\section{Ethics approval and consent to participate}

Not applicable.

\section{Funding}

This study was funded by Medical Health Science and Technology Project of Zhejiang Provincial Health Commission (No.2020KY561).

\section{References}

1. Bhan A, Soleimani M, Mandal SS: Long Noncoding RNA and Cancer: A New Paradigm. Cancer science. 2017; 77(15):3965-3981.

2. Sanchez Calle A, Kawamura Y: Emerging roles of long non-coding RNA in cancer. 2018; 109(7):2093-2100.

3. Huang Y, Guo Q, Ding XP, Wang X: Mechanism of long noncoding RNAs as transcriptional regulators in cancer. RNA biology. 2020:1-13.

4. Peng WX, Koirala P, Mo YY: LncRNA-mediated regulation of cell signaling in cancer. Oncogene. 2017; 36(41):5661-5667.

5. Yang G, Lu X, Yuan L: LncRNA: a link between RNA and cancer. Biochimica et biophysica acta. 2014; 1839(11):1097-1109. 
6. Yang R, Wang L, Han M: MNX1-AS1 is a novel biomarker for predicting clinical progression and poor prognosis in lung adenocarcinoma. Journal of cellular biochemistry. 2018.

7. Cheng Y, Pan Y, Pan Y, Wang O: MNX1-AS1 is a functional oncogene that induces EMT and activates the AKT/mTOR pathway and MNX1 in breast cancer. Cancer management and research. 2019; 11:803-812.

8. Ma JX, Yang YL, He XY, Pan XM, Wang Z, Qian YW: Long noncoding RNA MNX1-AS1 overexpression promotes the invasion and metastasis of gastric cancer through repressing CDKN1A. European review for medical and pharmacological sciences. 2019; 23(11):4756-4762.

9. Shuai Y, Ma Z, Liu W, Yu T, Yan C, Jiang H, Tian S, Xu T, Shu Y: TEAD4 modulated LncRNA MNX1-AS1 contributes to gastric cancer progression partly through suppressing BTG2 and activating BCL2. Molecular cancer. 2020; 19(1):6.

10. Zhang W, Huang L, Lu X, Wang K, Ning X, Liu Z: Upregulated expression of MNX1-AS1 long noncoding RNA predicts poor prognosis in gastric cancer. Bosnian journal of basic medical sciences. 2019; 19(2):164-171.

11. Lv Y, Li H, Li F, Liu P, Zhao X: Long Noncoding RNA MNX1-AS1 Knockdown Inhibits Cell Proliferation and Migration in Ovarian Cancer. Cancer biotherapy \& radiopharmaceuticals. 2017; 32(3):91-99.

12. Ji D, Wang Y, Sun B, Yang J, Luo X: Long non-coding RNA MNX1-AS1 promotes hepatocellular carcinoma proliferation and invasion through targeting miR-218-5p/COMMD8 axis. Biochemical and biophysical research communications. 2019; 513(3):669-674.

13. Wang J, Xing H, Nikzad AA, Liu B, Zhang Y, Li S, Zhang E, Jia Z: Long Noncoding RNA MNX1 antisense RNA 1 Exerts Oncogenic Functions in Bladder Cancer by Regulating miR-218-5p/RAB1A Axis. The Journal of pharmacology and experimental therapeutics. 2020; 372(3):237-247.

14. Chu J, Li H, Xing Y, Jia J, Sheng J, Yang L, Sun K, Qu Y, Zhang Y, Yin H et al: LncRNA MNX1-AS1 promotes progression of esophageal squamous cell carcinoma by regulating miR-34a/SIRT1 axis. Biomedicine \& pharmacotherapy = Biomedecine \& pharmacotherapie. 2019; 116:109029.

15. Fan E, Zhong Q, Feng G, Shuai Y, Wu M, Chen Q, Gou X, Chu J, Li H, Xing Y et al: LncRNA MNX1-AS1 promotes progression of esophageal squamous cell carcinoma by regulating miR-34a/SIRT1 axis. Human genomics. 2019; 116:109029.

16. Yang R, Wang L, Han M: MNX1-AS1 is a novel biomarker for predicting clinical progression and poor prognosis in lung adenocarcinoma. 2018.

17. Li Z, Wang F, Zhang S: Knockdown of IncRNA MNX1-AS1 suppresses cell proliferation, migration, and invasion in prostate cancer. FEBS open bio. 2019; 9(5):851-858.

18. Liu X, Yang Q, Yan J, Zhang X, Zheng M: LncRNA MNX1-AS1 promotes the progression of cervical cancer through activating MAPK pathway. Journal of cellular biochemistry. 2019; 120(3):4268-4277.

19. Tierney JF, Stewart LA, Ghersi D, Burdett S, Sydes MR: Practical methods for incorporating summary time-to-event data into meta-analysis. Trials. 2007; 8:16.

20. Liu G, Guo X, Zhang Y, Liu Y, Li D, Tang G, Cui S: Expression and significance of LncRNA MNX1-AS1 in non-small cell lung cancer. OncoTargets and therapy. 2019; 12:3129-3138.

21. Liu H, Han L, Liu Z, Gao N: Long noncoding RNA MNX1-AS1 contributes to lung cancer progression through the miR-527/BRF2 pathway. Journal of cellular physiology. 2019; 234(8):13843-13850.

22. Li AH, Zhang HH: Overexpression of IncRNA MNX1-AS1 is associated with poor clinical outcome in epithelial ovarian cancer. Journal of cellular biochemistry. 2017; 21(24):5618-5623.

23. Ye Y, Gu B, Wang Y, Shen S, Huang W: E2F1-mediated MNX1-AS1-miR-218-5p-SEC61A1 feedback loop contributes to the progression of colon adenocarcinoma. Journal of cellular biochemistry. 2019; 120(4):6145-6153.

24. Liu H, Han L, Liu Z, Gao N: Long noncoding RNA MNX1-AS1 contributes to lung cancer progression through the miR-527/BRF2 pathway. 2019; 234(8):13843-13850.

25. Gao Y, Xu Y, Wang J, Yang X, Wen L, Feng J: IncRNA MNX1-AS1 Promotes Glioblastoma Progression Through Inhibition of miR-4443. Oncology research. 2019; 27(3):341-347.

\section{Figures}




\section{4 records identified through database searching: PubMed $(n=19)$; Web of science $(n=21)$; CNKI $(n=3)$; Wanfang $(n=1)$.}

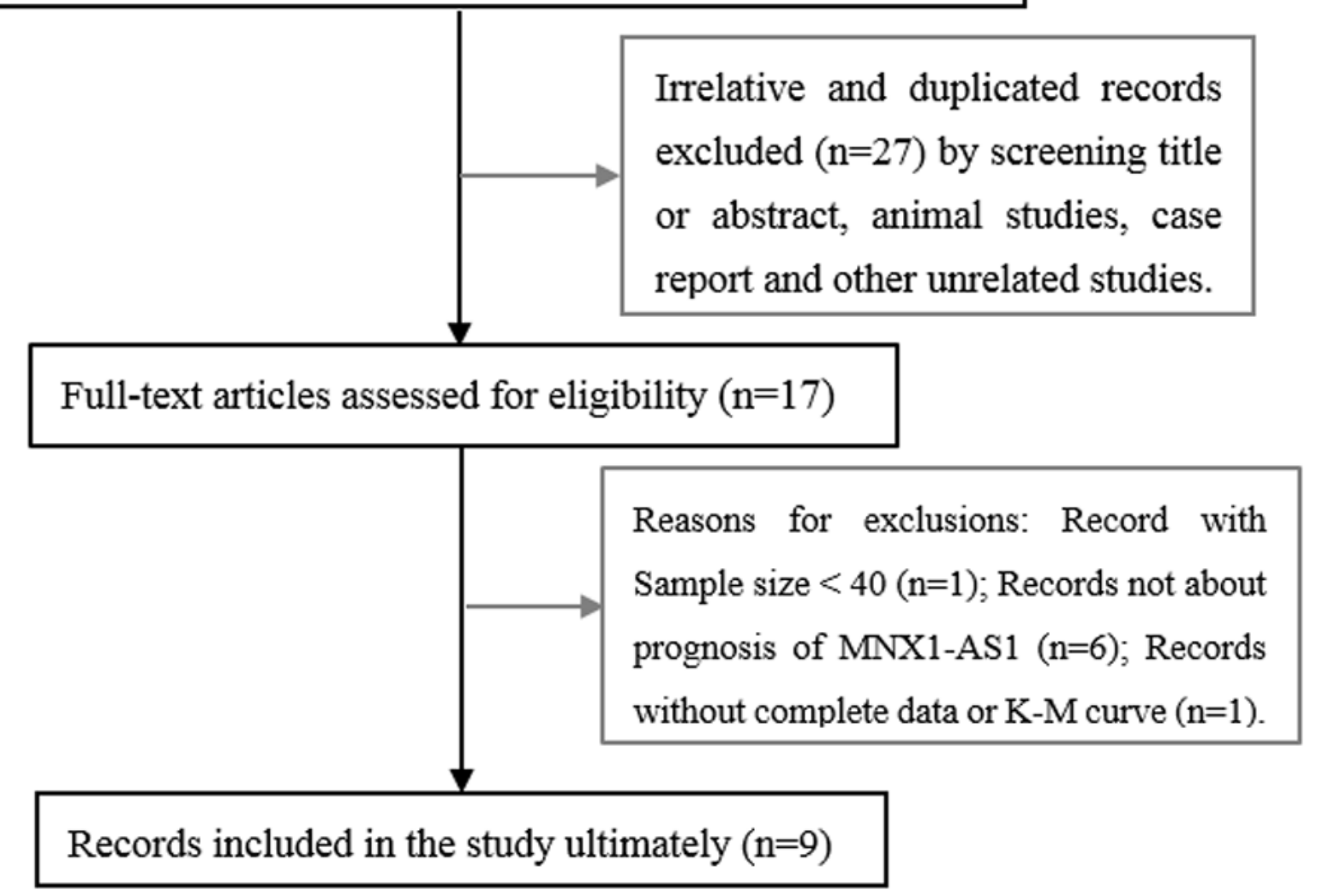

Figure 1

Flow diagram of the eligible articles.

A

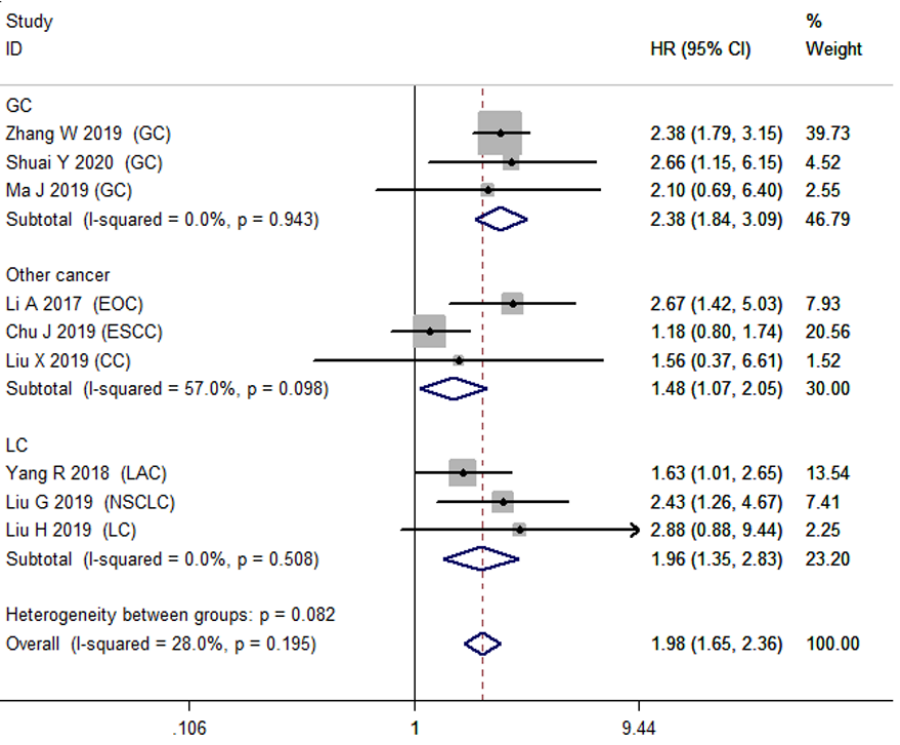

B

Study

ID $\quad H R(95 \% \mathrm{Cl})$ Weight

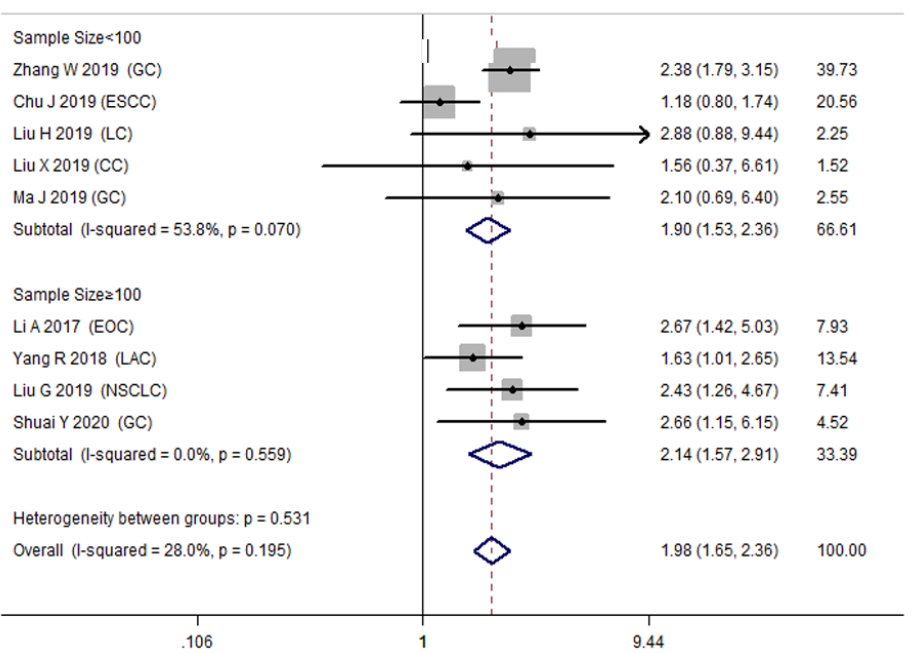

Figure 2

Forest plot of association of MNX1-AS1 expression with OS: (A) cancer type;(B) sample size. 
Funnel plot with pseudo 95\% confidence limits

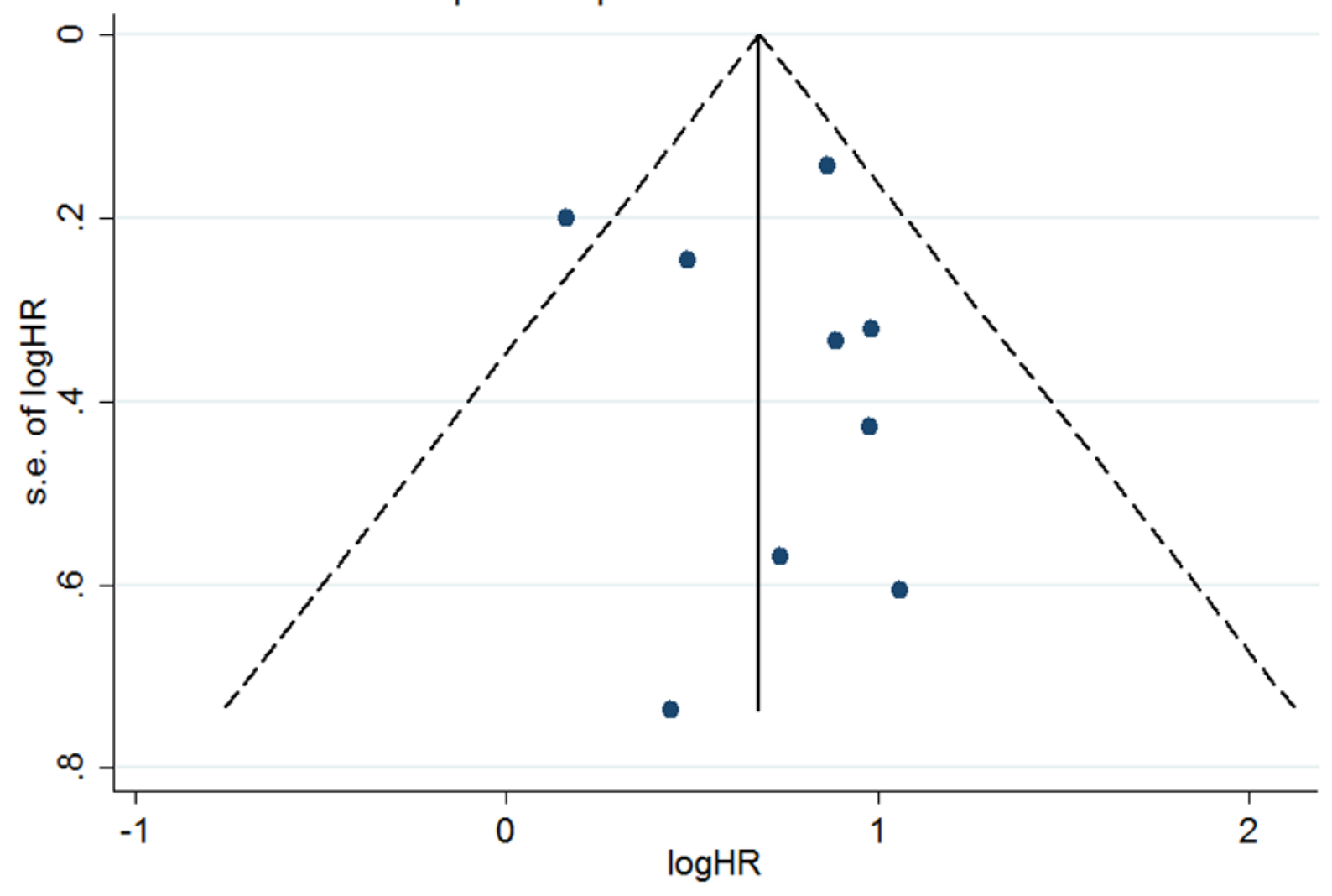

Figure 3

Funnel plot of OS.

\section{Supplementary Files}

This is a list of supplementary files associated with this preprint. Click to download.

- SupplementaryfileTableS1Thesummaryofpublishbias.docx 